\title{
Spectral gap, logarithmic Sobolev constant, and geometric bounds
}

\author{
Michel Ledoux
}

\begin{abstract}
We survey recent works on the connection between spectral gap and logarithmic Sobolev constants, and exponential integrability of Lipschitz functions. In particular, tools from measure concentration are used to describe bounds on the diameter of a (compact) Riemannian manifold and of Markov chains in terms of the first eigenvalue of the Laplacian and the logarithmic Sobolev constant. We examine similarly dimension free isoperimetric bounds using these parameters.
\end{abstract}

\section{Contents}

1. Introduction

2. Spectrum and exponential integrability

3. Spectral and diameter bounds

4. Logarithmic Sobolev constant and diameter bounds

5. Dimension free isoperimetric bounds

\section{Introduction}

In the recent years, it has been realized that simple measure theoretic arguments may be used to produce (sharp) bounds on geometric objects, such as the diameter of manifolds or graphs, in terms of spectral or logarithmic Sobolev constants. This question has of course a long run in Riemannian geometry and this work focuses more on the (elementary) methods than on the conclusions themselves. The key argument is described through the measure concentration and exponential integrability properties of distance functions under Poincaré or logarithmic Sobolev inequalities going back to the work of M. Gromov and V. Milman [G-M] and I. Herbst (cf. [Le5]). In particular, the approach avoids any type of purely geometric arguments and delicate heat kernel bounds, and produces bounds of the correct order of magnitude in the dimension. The investigation at the level of logarithmic Sobolev constants turns out to be of crucial interest in the study of rates of convergence to equilibrium, especially for Markov chains as developed by P. Diaconis and L. Saloff-Coste (cf. [D-SC], [SC3]). 
To describe the connection between spectral and logarithmic Sobolev inequalities, and integrability properties of distance functions, it will be convenient to use the language of metric measure spaces and of measure concentration (cf. [Le5]). Let thus $(X, d, \mu)$ be a metric measure space in the sense of [Grom2], that is $(X, d)$ is a metric space and $\mu$ is a finite non-negative Borel measure on $(X, d)$, normalized to be a probability measure $(\mu(X)=1)$. Define, for $\lambda \in \mathbb{R}$, the Laplace functional of $\mu$ on $(X, d)$ as

$$
\mathrm{E}_{(X, d, \mu)}(\lambda)=\sup \int_{X} \mathrm{e}^{\lambda F} d \mu
$$

where the supremum runs over all (bounded) 1-Lipschitz functions $F$ on $(X, d)$ such that $\int_{X} F d \mu=0$. By 1-Lipschitz, we understand that $|F(x)-F(y)| \leq d(x, y)$ for all $x, y \in X$. We often write more simply $\mathrm{E}_{\mu}=\mathrm{E}_{(X, d, \mu)}$. Note that $\mathrm{E}_{\mu}$ is an even function, non-decreasing on $[0, \infty)$. We will say, following [Grom2] (cf. [Le5]) that $(X, d, \mu)$ has exponential concentration whenever $\mathrm{E}_{(X, d, \mu)}\left(\lambda_{0}\right)<\infty$ for some $\lambda_{0}>0$, and that $(X, d, \mu)$ has normal concentration if for some constant $C>0$,

$$
\mathrm{E}_{(X, d, \mu)}(\lambda) \leq \mathrm{e}^{C \lambda^{2} / 2}, \quad \lambda \in \mathbb{R} .
$$

This terminology is motivated by the following elementary lemma that describes the geometric aspects, in terms of measure concentration, of these properties. For any two sets $A, B$ in $(X, d)$, set

$$
d(A, B)=\inf \{d(x, y) ; x \in A, y \in B\} .
$$

Lemma 1.1. For any two sets $A, B$ in $(X, d)$, and any $\lambda \geq 0$,

$$
\mu(A) \mu(B) \leq \mathrm{e}^{-\lambda d(A, B)} \mathrm{E}_{\mu}(\lambda)^{2} .
$$

In particular, if $(X, d, \mu)$ has normal concentration (with constant $C$ )

$$
\mu(A) \mu(B) \leq \mathrm{e}^{-d(A, B)^{2} / 4 C} .
$$

Proof. By definition of the Laplace functional $\mathrm{E}_{\mu}$ of $(X, d, \mu)$, for any (bounded) 1-Lipschitz function $F$ on $X$ and any $\lambda \geq 0$,

$$
\int_{A} \int_{B} \mathrm{e}^{\lambda[F(x)-F(y)]} d \mu(x) d \mu(y) \leq \int_{X} \mathrm{e}^{\lambda(F-M)} d \mu \int_{X} \mathrm{e}^{\lambda(M-F)} d \mu \leq \mathrm{E}_{\mu}(\lambda)^{2}
$$

where $M=\int_{X} F d \mu$. Choose now $F(x)=\min (d(x, B), r), x \in X$, so that

$$
\int_{A} \int_{B} \mathrm{e}^{\lambda[F(x)-F(y)]} d \mu(x) d \mu(y) \geq \mathrm{e}^{\lambda \min (d(A, B), r)} \mu(A) \mu(B) .
$$

Let then $r \rightarrow \infty$. If $(X, d, \mu)$ has normal concentration, optimize in $\lambda$. Lemma 1.1 is proved.

If $A$ is a subset of $(X, d)$, denote by

$$
A_{r}=\{x \in X ; d(x, A)<r\}
$$

its (open) neighborhood of order $r>0$.

Corollary 1.2. If $(X, d, \mu)$ has exponential concentration (respectively normal concentration), for every Borel set $A$ in $(X, d)$ such that $\mu(A) \geq \frac{1}{2}$,

$$
\mu\left(A_{r}\right) \geq 1-2 \mathrm{E}_{\mu}\left(\lambda_{0}\right)^{2} \mathrm{e}^{-\lambda_{0} r}
$$

(respectively

$$
\left.\mu\left(A_{r}\right) \geq 1-2 \mathrm{e}^{-r^{2} / 4 C}\right)
$$


for all $r>0$.

It is worthwhile mentioning for further comparison that whenever the diameter $D$ of $(X, d)$ is finite, $(X, d, \mu)$ has normal concentration with constant $D^{2}$ for any probability measure $\mu$ on the Borel sets of $(X, d)$. Indeed, let $F$ be a mean zero 1-Lipschitz function on $(X, d)$. By Jensen's inequality, for every $\lambda \in \mathbb{R}$,

$$
\int_{X} \mathrm{e}^{\lambda F} d \mu \leq \int_{X} \int_{X} \mathrm{e}^{\lambda[F(x)-F(y)]} d \mu(x) d \mu(y) \leq \sum_{i=0}^{\infty} \frac{(D \lambda)^{2 i}}{(2 i) !} \leq \mathrm{e}^{D^{2} \lambda^{2} / 2} .
$$

The claim follows.

In these notes, we survey recent developments on spectral and logarithmic Sobolev bounds by the preceding measure concentration tools, mainly taken from the references [SC2], [D-SC], [Le3]. In Section 2, we show how the existence of a spectral gap, or Poincaré inequality, implies exponential concentration. This observation turns out to have rather useful consequences to bounds on the diameter to which we turn next in Section 3. All these results have analogous counterparts on graphs and discrete structures. In Section 4, we develop the corresponding investigation at the level of logarithmic Sobolev inequalities that provide more precise bounds. Namely, together with the Herbst argument, we show how the logarithmic Sobolev constant entails normal concentration of Lipschitz functions and deduce then sharp bounds on the diameter. Again, the discrete case leads to a number of considerations of interest in connection with geometric bounds. We briefly discuss the analogous conclusions under the entropic constant. In the last part, we investigate isoperimetric bounds under spectral and logarithmic Sobolev constants using some simple semigroup tools, and describe, as a main feature, dimension free inequalities of isoperimetric type.

\section{Spectrum and exponential integrability}

Assume first we are given a smooth complete connected Riemannian manifold $(X, g)$ (without boundary) with Riemannian metric $g$ and finite volume $V$. Denote by $d$ the distance function induced by $g$ on $X$ and by $d \mu=\frac{d v}{V}$ the normalized Riemannian volume element on $(X, g)$. Let furthermore $\lambda_{1}=\lambda_{1}(X)$ be the first non-trivial eigenvalue of the Laplacian $\Delta_{g}$ on $X$. By the Raleigh-Ritz variational principle (cf. [Cha1], [G-H-L] ...), $\lambda_{1}$ is characterized by the spectral gap, or Poincaré, inequality

$$
\lambda_{1} \int_{X} f^{2} d \mu \leq \int_{X} f\left(-\Delta_{g} f\right) d \mu=\int_{X}|\nabla f|^{2} d \mu
$$

for all smooth real-valued functions $f$ on $(X, g)$ such that $\int_{X} f d \mu=0$, where $|\nabla f|$ denotes the Riemannian length of the gradient of $f$.

The following result goes back independently to M. Gromov and V. Milman [G-M] (in a geometric context) and A. Borovkov and S. Utev [B-U] (in a probabilistic context). (See also $[\mathrm{Br}]$.) It has been investigated in [A-M-S] and [A-S] using moment bounds, and in [Sc] using a differential inequality on Laplace transforms (similar to the Herbst argument presented in Section 4). We follow here the approach by S. Aida and D. Stroock [A-S].

THEOREM 2.1. Let $(X, g)$ be a smooth complete connected Riemannian manifold with finite volume and normalized Riemannian measure $\mu$. Denote by $\lambda_{1}=\lambda_{1}(X)$ 
the first non-trivial eigenvalue of the Laplacian $\Delta_{g}$ on $(X, g)$. Then,

$$
\mathrm{E}_{(X, d, \mu)}\left(\sqrt{\lambda}_{1}\right) \leq 3 .
$$

In particular $(X, d, \mu)$ has exponential concentration whenever $\lambda_{1}>0$.

Proof. Set $u(\lambda)=\int_{X} \mathrm{e}^{\lambda F} d \mu, \lambda \geq 0$, where $F$ is bounded and such that $\int_{X} F d \mu=0$. Since $F$ may be assumed smooth enough, we can have that $|\nabla F| \leq 1$ everywhere. Apply (2.1) to $f=\mathrm{e}^{\lambda F / 2}, \lambda \geq 0$. Since

$$
\int_{X}|\nabla f|^{2} d \mu=\frac{\lambda^{2}}{4} \int_{X}|\nabla F|^{2} \mathrm{e}^{\lambda F} d \mu \leq \frac{\lambda^{2}}{4} \int_{X} \mathrm{e}^{\lambda F} d \mu,
$$

we get that

$$
u(\lambda)-u\left(\frac{\lambda}{2}\right)^{2} \leq \frac{\lambda^{2}}{4 \lambda_{1}} u(\lambda) .
$$

Hence, for every $\lambda<2 \sqrt{\lambda_{1}}$,

$$
u(\lambda) \leq \frac{1}{1-\lambda^{2} / 4 \lambda_{1}} u\left(\frac{\lambda}{2}\right)^{2} .
$$

Applying the same inequality for $\lambda / 2$ and iterating, yields, after $n$ steps,

$$
u(\lambda) \leq \prod_{k=0}^{n-1}\left(\frac{1}{1-\lambda^{2} / 4^{k+1} \lambda_{1}}\right)^{2^{k}} u\left(\frac{\lambda}{2^{n}}\right)^{2^{n}} .
$$

Since $u(\lambda)=1+o(\lambda)$, we have that $u\left(\lambda / 2^{n}\right)^{2^{n}} \rightarrow 1$ as $n \rightarrow 0$. Therefore,

$$
u(\lambda) \leq \prod_{k=0}^{\infty}\left(\frac{1}{1-\lambda^{2} / 4^{k+1} \lambda_{1}}\right)^{2^{k}}
$$

where the infinite product converges whenever $\lambda<2 \sqrt{\lambda_{1}}$. Setting for example $\lambda=\sqrt{\lambda_{1}}$ yields that

$$
\int_{X} \mathrm{e}^{\sqrt{\lambda_{1}} F} d \mu=u\left(\sqrt{\lambda_{1}}\right) \leq 3
$$

The proof of Theorem 2.1 is complete.

It is a simple yet non-trivial observation that $\lambda_{1}(X \times Y)=\min \left(\lambda_{1}(X), \lambda_{1}(Y)\right)$ for Riemannian manifolds $X$ and $Y$. Theorem 2.1 therefore provides a useful tool to concentration in product spaces (cf. [Le5]).

Theorem 2.1 has an analogue on graphs to which we turn now. It is convenient to deal with finite state Markov chains.

Let $X$ be a finite (or countable) set. Let $\Pi(x, y) \geq 0, x, y \in X$, satisfy

$$
\sum_{y \in X} \Pi(x, y)=1
$$

for every $x \in X$. Assume furthermore that there is a symmetric invariant probability measure $\mu$ on $X$ for $\Pi$, that is $\Pi(x, y) \mu(\{x\})$ is symmetric in $x$ and $y$ and $\sum_{x} \Pi(x, y) \mu(\{x\})=\mu(\{y\})$ for every $y \in X$. In other words, $(\Pi, \mu)$ is a reversible Markov chain (cf. e.g. [SC3] and the references therein). Define, for $f, g: X \rightarrow \mathbb{R}$ say finitely supported, the Dirichlet form

$$
\mathcal{Q}(f, g)=\sum_{x, y \in X}[f(x)-f(y)][g(x)-g(y)] \Pi(x, y) \mu(\{x\}) .
$$


We may speak of the spectral gap, or the Poincaré constant, of the chain $(\Pi, \mu)$ as the largest $\lambda_{1}$ such that for all $f$ 's (with finite support) such that $\int_{X} f d \mu=0$,

$$
\lambda_{1} \int_{X} f^{2} d \mu \leq \mathcal{Q}(f, f) .
$$

Set also

$$
\|\left.|f|\right|_{\infty} ^{2}=\sup _{x \in X} \sum_{y \in X}|f(x)-f(y)|^{2} \Pi(x, y) .
$$

The triple norm $\left|\|\cdot \mid\|_{\infty}\right.$ may be thought of as a discrete version of the Lipschitz norm in the continuous setting. Although it may not be well adapted to all discrete structures, it behaves similarly for what concerns spectrum and exponential concentration. Equip $X$ with the distance associated with ||$|\cdot| \|\left.\right|_{\infty}$ defined as

$$
d_{\mathcal{Q}}(x, y)=\sup _{\|f\|_{\infty} \leq 1}[f(x)-f(y)], \quad x, y \in X .
$$

Theorem 2.2 below is the analogue of Theorem 2.1 in this discrete setting (cf. [A-S]). The proof is essentially the same.

THEOREM 2.2. Let $(\Pi, \mu)$ be a reversible Markov chain on $X$ as before with spectral gap $\lambda_{1}$. Then

$$
\mathrm{E}_{\left(X, d_{\mathcal{Q}}, \mu\right)}\left(\sqrt{\lambda_{1} / 2}\right) \leq 3
$$

In particular $\left(X, d_{\mathcal{Q}}, \mu\right)$ has exponential concentration whenever $\lambda_{1}>0$.

Proof. We proceed as for Theorem 2.1. The main observation is that, for every $F$ on $X$ and every $\lambda \geq 0$,

$$
\mathcal{Q}\left(\mathrm{e}^{\lambda F / 2}, \mathrm{e}^{\lambda F / 2}\right) \leq \frac{1}{2}\|\| F \|_{\infty}^{2} \lambda^{2} \int_{X} \mathrm{e}^{\lambda F} d \mu .
$$

Indeed, by symmetry,

$$
\begin{aligned}
\mathcal{Q}\left(\mathrm{e}^{\lambda F / 2}, \mathrm{e}^{\lambda F / 2}\right) & =\sum_{x, y \in X}\left[\mathrm{e}^{\lambda F(x) / 2}-\mathrm{e}^{\lambda F(y) / 2}\right]^{2} \Pi(x, y) \mu(\{x\}) \\
& =2 \sum_{F(y)<F(x)}\left[\mathrm{e}^{\lambda F(x) / 2}-\mathrm{e}^{\lambda F(y) / 2}\right]^{2} \Pi(x, y) \mu(\{x\}) \\
& \leq \frac{\lambda^{2}}{2} \sum_{x, y \in X}[F(x)-F(y)]^{2} \mathrm{e}^{\lambda F(x)} \Pi(x, y) \mu(\{x\})
\end{aligned}
$$

from which (2.3) follows by definition of $\||F|\|_{\infty}$.

Assume now that $F$ is bounded with mean zero and $\||F|\|_{\infty} \leq 1$. Set $u(\lambda)=$ $\int_{X} \mathrm{e}^{\lambda F} d \mu, \lambda \geq 0$. Applying (2.2) to $\mathrm{e}^{\lambda F / 2}$ yields, together with (2.3),

$$
u(\lambda)-u\left(\frac{\lambda}{2}\right)^{2} \leq \frac{\lambda^{2}}{2 \lambda_{1}} u(\lambda),
$$

that is, for every $0 \leq \lambda<\sqrt{2 \lambda_{1}}$,

$$
u(\lambda) \leq \frac{1}{1-\lambda^{2} / 2 \lambda_{1}} u\left(\frac{\lambda}{2}\right)^{2} .
$$

We then conclude as for Theorem 2.1, with $\lambda_{1}$ replaced by $\lambda_{1} / 2$. 
The distance most often used in such contexts is however not $d_{\mathcal{Q}}$ but the combinatoric distance $d_{c}$ associated with the graph with vertex-set $X$ and edge-set $\{(x, y): \Pi(x, y)>0\}$. This distance can be defined as the minimal number of edges one has to cross to go from $x$ to $y$. Equivalently,

$$
d_{c}(x, y)=\sup _{\|\nabla f\|_{\infty} \leq 1}[f(x)-f(y)]
$$

where

$$
\|\nabla f\|_{\infty}=\sup \{|f(x)-f(y)| ; \Pi(x, y)>0\} .
$$

Now since $\sum_{y} \Pi(x, y)=1$,

$$
\|f\|_{\infty}^{2} \leq\|\nabla f\|_{\infty}^{2}
$$

In particular $d_{c} \leq d_{\mathcal{Q}}$ so that Theorem 2.2 also holds for the metric measure space $\left(X, d_{c}, \mu\right)$.

As an example, let $X=(V, \mathcal{E})$ be a finite connected graph with set of vertices $V$ and symmetric set of edges $\mathcal{E}$. Equip $V$ with the normalized uniform measure $\mu$ and the graph distance $d_{c}$. We may consider $\Pi(x, y)=\frac{1}{k(x)}$ whenever $x$ and $y$ are adjacent in $V$ and 0 otherwise where $k(x)$ is the number of neighbors of $x$. Consider the quadratic form

$$
\widetilde{\mathcal{Q}}(f, f)=\sum_{x \sim y}[f(x)-f(y)]^{2}
$$

where the sum runs over all neighbors $x \sim y$ in $X$. Since $X$ is connected, $\widetilde{\mathcal{Q}}(f, f) \geq 0$ for all $f$ 's and is zero whenever $f$ is constant. Let $\lambda_{1}>0$ be the first non-trivial eigenvalue of $\widetilde{\mathcal{Q}}$ (that is of the Laplace operator on $X$ ). As a consequence of Theorem 2.2, we have the following result.

Corollary 2.3. Let $k_{0}=\max \{k(x) ; x \in V\}<\infty$. Then

$$
\mathrm{E}_{(X, d, \mu)}\left(\sqrt{\frac{\lambda_{1}}{2 k_{0}}}\right) \leq 3
$$

The most important examples of applications of the preceding corollary are the Cayley graphs. If $V$ is a finite group and $S \subset V$ a symmetric set of generators of $V$, we may join $x$ and $y$ in $V$ by an edge if $x=s^{-1} y$ for some $s \in S$. The path distance on $X=(V, \mathcal{E})$ is the word distance in $V$ induced by $S$ and $k_{0}=\operatorname{Card}(S)$. For oriented graphs, see $[\mathrm{Al}],[\mathrm{A}-\mathrm{M}]$.

To conclude this section, we show, on the basis of Lemma 1.1, how the preceding exponential integrability results may be applied to bounds on the spectral gap $\lambda_{1}$ in terms of distances between disjoints sets. Letting indeed $\lambda_{0}=\sqrt{\lambda_{1}}$ in Lemma 1.1, it follows from Theorem 2.1 that, for every sets $A, B$ in $X$,

$$
\lambda_{1} \leq \frac{1}{d(A, B)^{2}} \log ^{2}\left(\frac{C}{\mu(A) \mu(B)}\right)
$$

with $C=9$. (In the context of Theorem 2.2 , replace $\lambda_{1}$ by $\lambda_{1} / 2$.) As discussed in [Bo-L], the preceding arguments may easily be improved to reach $C=1$ in (2.4). Inequalities such as (2.4) have been considered by F. R. K. Chung, A. Grigory'an and S.-T. Yau [C-G-Y1] who showed (2.4) (with $C=4$ ) using heat kernel expansions, and then using the wave equation [C-G-Y2] (with $C=\mathrm{e}$ ). They actually establish similar inequalities for the all sequence of eigenvalues, something not considered here. They also establish similar results on graphs. 


\section{Spectral and diameter bounds}

On the basis of the results of the preceding section, we investigate here some relationships between spectral and diameter bounds. The following result is taken from [Le5], and may be traced back in the work by R. Brooks $[\mathrm{Br}]$.

Assume that we are given a smooth complete connected Riemannian manifold $(X, g)$, (without boundary), not necessarily compact but with finite volume $V$. Denote by $d \mu=\frac{d v}{V}$ the normalized Riemannian volume element. Let as before $\lambda_{1}=\lambda_{1}(X)$ be the first non-trivial eigenvalue of the Laplace operator $\Delta_{g}$ on $(X, g)$.

If $B(x, r)$ is the (open) ball with center $x$ and radius $r>0$ in $X$, it follows from Theorem 2.1 together with Corollary 1.2 that $\lambda_{1}=\lambda_{1}(X)=0$ as soon as

$$
\limsup _{r \rightarrow \infty} \frac{1}{r} \log (1-\mu(B(x, r)))=0
$$

for some (all) $x$ in $X$ (cf. [Br]). The following is a kind of converse.

TheOREM 3.1. Let $(X, g)$ be a smooth complete connected Riemannian manifold with dimension $n$ and finite volume. Let $\mu$ be the normalized Riemannian volume on $(X, g)$. Assume that the Ricci curvature of $(X, g)$ is bounded below. Then $(X, g)$ is compact as soon as

$$
\liminf _{r \rightarrow \infty} \frac{1}{r} \log (1-\mu(B(x, r)))=-\infty
$$

for some (or all) $x \in X$. In particular $\lambda_{1}=\lambda_{1}(X)>0$ under this condition. Furthermore, if $(X, g)$ has non-negative Ricci curvature and if $D$ is the diameter of $X$, then

$$
\lambda_{1} \leq \frac{C_{n}}{D^{2}}
$$

where $C_{n}>0$ only depends on the dimension $n$ of $X$.

The upper bound (3.2) goes back to the work by S.-Y. Cheng [Chen] in Riemannian geometry (see also [Cha1], [L-Y1] and below). In the opposite direction, it has been shown by P. Li [Li] and H. C. Yang and J. Q. Zhong [Y-Z] that when $(X, g)$ has non-negative Ricci curvature,

$$
\lambda_{1} \geq \frac{\pi^{2}}{D^{2}}
$$

This lower bound is optimal since achieved on the one-dimensional torus.

Proof of Theorem 3.1. We proceed by contradiction and assume that $X$ is not compact. Choose $B\left(x, r_{0}\right)$ a geodesic ball in $X$ with center $x$ and radius $r_{0}>0$ such that $\mu\left(B\left(x, r_{0}\right)\right) \geq \frac{1}{2}$. By non-compactness (and completeness), for every $r>$ 0 , we can take $z$ at distance $r_{0}+2 r$ from $x$. In particular, $B\left(x, r_{0}\right) \subset B\left(z, 2\left(r_{0}+r\right)\right)$. By the Riemannian volume comparison theorem [C-E], [Cha2], for every $y \in X$ and $0<s<t$,

$$
\frac{\mu(B(y, t))}{\mu(B(y, s))} \leq\left(\frac{t}{s}\right)^{n} \mathrm{e}^{t \sqrt{(n-1) K}}
$$

where $-K, K \geq 0$, is the lower bound on the Ricci curvature of $(X, g)$. Therefore,

$$
\begin{aligned}
\mu(B(z, r)) & \geq\left(\frac{r}{2\left(r_{0}+r\right)}\right)^{n} \mathrm{e}^{-2\left(r+r_{0}\right) \sqrt{(n-1) K}} \mu\left(B\left(\left(z, 2\left(r_{0}+r\right)\right)\right)\right. \\
& \geq \frac{1}{2}\left(\frac{r}{2\left(r_{0}+r\right)}\right)^{n} \mathrm{e}^{-2\left(r_{0}+r\right) \sqrt{(n-1) K}}
\end{aligned}
$$


where we used that $\mu\left(B\left(z, 2\left(r_{0}+r\right)\right)\right) \geq \mu\left(B\left(x, r_{0}\right)\right) \geq \frac{1}{2}$. Since $B(z, r)$ is included into the complement of $B\left(x, r_{0}+r\right)$,

$$
1-\mu\left(B\left(x, r+r_{0}\right)\right) \geq \frac{1}{2}\left(\frac{r}{2\left(r_{0}+r\right)}\right)^{n} \mathrm{e}^{-2\left(r_{0}+r\right) \sqrt{(n-1) K}}
$$

which is impossible as $r \rightarrow \infty$ by the assumption. The first part of the theorem is established.

Thus $(X, g)$ is compact. Denote by $D$ its diameter. Assume that $(X, g)$ has non-negative Ricci curvature. That is, we may take $K=0$ in (3.4) and (3.5). By Theorem 2.1 together with Corollary 1.2, for every measurable subset $A$ in $X$ such that $\mu(A) \geq \frac{1}{2}$, and every $r>0$,

$$
1-\mu\left(A_{r}\right) \leq 18 \mathrm{e}^{-\sqrt{\lambda_{1}} r} .
$$

We distinguish between two cases. If $\mu\left(B\left(x, \frac{D}{8}\right)\right) \geq \frac{1}{2}$, apply (3.6) to $A=B\left(x, \frac{D}{8}\right)$. By definition of $D$, we may choose $r=r_{0}=\frac{D}{8}$ in (3.5) to get

$$
\frac{1}{2 \cdot 4^{n}} \leq 1-\mu\left(A_{D / 8}\right) \leq 18 \mathrm{e}^{-\sqrt{\lambda_{1}} D / 8} .
$$

If $\mu\left(B\left(x, \frac{D}{8}\right)\right)<\frac{1}{2}$, apply (3.6) to $A$ the complement of $B\left(x, \frac{D}{8}\right)$. Since the ball $B\left(x, \frac{D}{16}\right)$ is included into the complement of $A_{D / 16}$ and since by (3.4) with $t=D$,

$$
\mu\left(B\left(x, \frac{D}{16}\right)\right) \geq \frac{1}{16^{n}},
$$

we get from (3.6) with $r=\frac{D}{16}$ that

$$
\frac{1}{16^{n}} \leq 1-\mu\left(A_{D / 16}\right) \leq 18 \mathrm{e}^{-\sqrt{\lambda_{1}} D / 16} .
$$

The conclusion easily follows from either case, with a constant $C_{n}$ of the order of $n^{2}$ as $n$ is large. Theorem 3.1 is established.

Analogous conclusions may be obtained in the discrete case. Let as before $\Pi(x, y)$ be a Markov chain on a finite state space $X$ with symmetric invariant probability measure $\mu$. Recall $\lambda_{1}$ the spectral gap of $(\Pi, \mu)$ and $d_{\mathcal{Q}}$ the distance defined from the norm

$$
\||f|\|_{\infty}^{2}=\sup _{x \in X} \sum_{y \in X}|f(x)-f(y)|^{2} \Pi(x, y) .
$$

Denote by $D_{\mathcal{Q}}$ the diameter of $X$ for $d_{\mathcal{Q}}$.

Proposition 3.2. If $\mu$ is nearly constant, that is if there exists $C>0$ such that, for every $x, \mu(\{x\}) \leq C \min _{y \in X} \mu(\{y\})$, then

$$
D_{\mathcal{Q}}^{2} \leq \frac{(4 \log (3 C|X|))^{2}}{\lambda_{1}}
$$

where $|X|$ is the cardinal of $X$.

Proof. Consider two points $x, y \in X$ such that $d(x, y)=D_{\mathcal{Q}}$. By Lemma 1.1 and Theorem 2.2,

$$
\mu(\{x\}) \mu(\{y\}) \leq 9 \mathrm{e}^{-D_{\mathcal{Q}} \sqrt{\lambda_{1}} / 2} .
$$

Since, by the hypothesis on $\mu, \min _{z \in X} \mu(\{z\}) \geq(C|X|)^{-1}$, the conclusion follows. 
Recall that the combinatoric diameter $D_{c}$ is less than or equal to $D_{\mathcal{Q}}$. As such, Proposition 3.2 goes back to $[\mathrm{A}-\mathrm{M}]$ (see also [Al], [Chu]), where it is observed that the bound on $D_{c}$ of Proposition 3.2 is optimal on the class of regular graphs.

\section{Logarithmic Sobolev constant and diameter bounds}

In this section, we turn to the corresponding investigation for logarithmic Sobolev inequalities. Logarithmic Sobolev constants actually provide sharper bounds than spectral gaps, and are of importance in the study of rates of convergence to equilibrium.

To start with, let as before $(X, g)$ be a complete connected Riemannian manifold (without boundary) with finite volume $V$. Let $d$ be the distance function associated to $g$ and $d \mu=\frac{d v}{V}$ the normalized volume element. In analogy with the first nontrivial eigenvalue $\lambda_{1}=\lambda_{1}(X)$ of $\Delta_{g}$ on $X$, define the logarithmic Sobolev constant $\rho_{0}=\rho_{0}(X)$ of $\Delta_{g}$ as the largest constant $\rho$ such that for every smooth function $f$ on $X$ with $\int_{X} f^{2} d \mu=1$,

$$
\rho \int_{X} f^{2} \log f^{2} d \mu \leq 2 \int_{X} f\left(-\Delta_{g} f\right) d \mu=2 \int_{X}|\nabla f|^{2} d \mu .
$$

Applying (4.1) to $1+\varepsilon f$, a simple Taylor expansion as $\varepsilon \rightarrow 0$ shows that $\lambda_{1} \geq \rho_{0}$.

Note that, as for $\lambda_{1}=\lambda_{1}(X)$, one may show (cf. [Gros], [Le3]) that $\rho_{0}(X \times Y)=$ $\min \left(\rho_{0}(X), \rho_{0}(Y)\right)$ for Riemannian manifolds $X$ and $Y$.

It is a non-trivial result, due to O. Rothaus [Ro1], that whenever $X$ is compact,

$$
\lambda_{1} \geq \rho_{0}>0 \text {. }
$$

When the Ricci curvature of $(X, g)$ is uniformly bounded below by a strictly positive constant $R$, it goes back to A. Lichnerowicz (cf. [Cha1], [G-H-L]) that $\lambda_{1} \geq R_{n}$ where $R_{n}=\frac{R}{1-\frac{1}{n}}$, with equality if and only if $X$ is a sphere (Obata's theorem). This lower bound has been shown to hold similarly for the logarithmic Sobolev constant by D. Bakry and M. Emery [B-E] (cf. [Ba]) so that

$$
\lambda_{1} \geq \rho_{0} \geq R_{n}
$$

The case of equality for $\rho_{0}$ is a consequence of Obata's theorem due to an improvement of the preceding by O. Rothaus [Ro2] who showed that when $(X, g)$ is compact and $\mathrm{Ric}_{g} \geq R(R \in \mathbb{R})$,

$$
\rho_{0} \geq \alpha_{n} \lambda_{1}+\left(1-\alpha_{n}\right) R_{n}
$$

where $\alpha_{n}=4 n /(n+1)^{2}$. In particular, $\lambda_{1}$ and $\rho_{0}$ are of the same order if $(X, g)$ has non-negative Ricci curvature. As examples, $\rho_{0}=\lambda_{1}=n$ on the $n$-sphere [M-W]. On the $n$-dimensional torus, $\lambda_{1}=\rho_{0}=1$. The question whether $\rho_{0}<\lambda_{1}$ in this setting has been open for some time until the geometric investigation by L. Saloff-Coste [SC2]. He actually proved, using heat kernel bounds and equilibrium rates, that the existence of a logarithmic Sobolev inequality in a Riemannian manifold with finite volume and Ricci curvature bounded from below forces the manifold to be compact. It is known that there exist non-compact manifolds of finite volume with $\lambda_{1}>0$. In particular, there exist compact manifolds of constant negative sectional curvature with spectral gaps uniformly bounded away from zero, and arbitrarily large diameters (cf. [SC2]. This yield examples for which the ratio $\rho_{0} / \lambda_{1}$ can be made arbitrarily small. 
We present below a simplified argument of this result based on Theorem 3.1 and normal concentration. To this task, we develop, for the logarithmic Sobolev inequality, the connection with exponential integrability and measure concentration as for the spectral inequalities in Sections 2 and 3. This will be achieved by the Herbst argument from a logarithmic Sobolev inequality to exponential integrability. It goes back to an unpublished argument by I. Herbst [Da-S], revived in the past years by S. Aida, T. Masuda and I. Shigekawa [A-M-S]. Relevance to measure concentration was emphasized in [Le2], and further developed in [Le3] (cf. [Le5] for the historical developments).

The principle is similar to the application of spectral properties to concentration presented in Section 2.1, but logarithmic Sobolev inequalities allow us to reach normal concentration. Let $F$ be a smooth bounded 1-Lipschitz function on $X$ such that $\int_{X} F d \mu=0$. In particular, since $F$ is assumed to be regular enough, we can have that $|\nabla F| \leq 1$ at every point. We apply (4.1) to $f^{2}=\mathrm{e}^{\lambda F}$ for every $\lambda \in \mathbb{R}$. We have

$$
\int_{X}|\nabla f|^{2} d \mu=\frac{\lambda^{2}}{4} \int_{X}|\nabla F|^{2} \mathrm{e}^{\lambda F} d \mu \leq \frac{\lambda^{2}}{4} \int_{X} \mathrm{e}^{\lambda F} d \mu .
$$

Setting $u(\lambda)=\int_{X} \mathrm{e}^{\lambda F} d \mu, \lambda \in \mathbb{R}$, by the definition of entropy,

$$
\lambda u^{\prime}(\lambda)-u(\lambda) \log u(\lambda) \leq \frac{1}{2 \rho_{0}} \lambda^{2} u(\lambda) .
$$

In other words, if $U(\lambda)=\frac{1}{\lambda} \log u(\lambda), U(0)=\int_{X} F d \mu=0$, then

$$
U^{\prime}(\lambda) \leq \frac{1}{2 \rho_{0}}, \quad \lambda \in \mathbb{R}
$$

Therefore $U(\lambda) \leq \frac{\lambda}{2 \rho_{0}}$ from which we immediately conclude that

$$
u(\lambda)=\int_{X} \mathrm{e}^{\lambda F} d \mu \leq \mathrm{e}^{\lambda^{2} / 2 \rho_{0}}
$$

for every $\lambda \in \mathbb{R}$.

We summarize the preceding argument in the following statement. Recall the Laplace functional $\mathrm{E}_{(X, d, \mu)}$ of $\mu$ on $X$.

TheOREM 4.1. Let $(X, g)$ be a smooth complete connected Riemannian manifold with finite volume and normalized Riemannian measure $\mu$. Denote by $\rho_{0}$ the logarithmic Sobolev constant of $\Delta_{g}$ on $(X, g)$. Then,

$$
\mathrm{E}_{(X, d, \mu)}(\lambda) \leq \mathrm{e}^{\lambda^{2} / 2 \rho_{0}}, \quad \lambda \in \mathbb{R} .
$$

In particular, $(X, d, \mu)$ has normal concentration whenever $\rho_{0}>0$.

As announced, it was shown by L. Saloff-Coste [SC2] that the existence of $\rho_{0}=$ $\rho_{0}(X)>0$ forces a Riemannian manifold with finite volume and Ricci curvature bounded from below to be compact. Together with Theorem 4.1, we may present, along the lines of the proof of Theorem 3.1, a sharp improvement of the quantitative bound on the diameter of $X$ in terms of the logarithmic Sobolev constant $\rho_{0}$.

THEOREM 4.2. Let $(X, g)$ be a smooth complete connected Riemannian manifold with dimension $n$ and finite volume. Let $\mu$ be the normalized Riemannian volume element on $(X, g)$ and denote by $\rho_{0}=\rho_{0}(X)$ the logarithmic Sobolev constant of $\Delta_{g}$ on $(X, g)$. Assume that $\operatorname{Ric}_{g} \geq-K, K \geq 0$. If $\rho_{0}>0$, then $(X, g)$ is compact. 
Furthermore, if $D$ is the diameter of $X$, there exists a numerical constant $C>0$ such that

$$
D \leq C \sqrt{n} \max \left(\frac{\sqrt{K}}{\rho_{0}}, \frac{1}{\sqrt{\rho}_{0}}\right) .
$$

It is known from the theory of hypercontractive semigroups (cf. [De-S]) that conversely there exists $C(n, K, \varepsilon)$ such that

$$
\rho_{0} \geq \frac{C(n, K, \varepsilon)}{D}
$$

whenever $\lambda_{1} \geq \varepsilon>0$.

Proof. By Theorem 4.1 and Corollary 1.2,

$$
1-\mu\left(A_{r}\right) \leq 2 \mathrm{e}^{-\rho_{0} r^{2} / 4}
$$

for every $r>0$ and $A \subset X$ such that $\mu(A) \geq \frac{1}{2}$. It is thus clear that

$$
\liminf _{r \rightarrow \infty} \frac{1}{r} \log (1-\mu(B(x, r)))=-\infty
$$

so that $(X, g)$ is compact by Theorem 3.1. To establish the bound on the diameter $D$, we repeat the proof of Theorem 3.1, replacing (3.6) by (4.5). Let $B\left(x, \frac{D}{8}\right)$ be the ball with center $x$ and radius $\frac{D}{8}$. We distinguish between two cases. If $\mu\left(B\left(x, \frac{D}{8}\right)\right) \geq \frac{1}{2}$, apply $(4.5)$ to $A=B\left(x, \frac{D}{8}\right)$. By definition of $D$, we may choose $r=r_{0}=\frac{D}{8}$ in (3.5) to get

$$
\frac{1}{2} \cdot \frac{1}{4^{n}} \mathrm{e}^{-\sqrt{(n-1) K} D / 2} \leq 1-\mu\left(A_{D / 8}\right) \leq 2 \mathrm{e}^{-\rho_{0} D^{2} / 256} .
$$

If $\mu\left(B\left(x, \frac{D}{8}\right)\right)<\frac{1}{2}$, apply (4.5) to $A$ the complement of $B\left(x, \frac{D}{8}\right)$. Since the ball $B\left(x, \frac{D}{16}\right)$ is included into the complement of $A_{D / 16}$ and since by (3.4)

$$
\mu\left(x_{0}, \frac{D}{16}\right) \geq \frac{1}{16^{n}} \mathrm{e}^{-\sqrt{(n-1) K} D},
$$

it follows from (4.5) with $r=\frac{D}{16}$ that

$$
\frac{1}{16^{n}} \mathrm{e}^{-\sqrt{(n-1) K} D} \leq 1-\mu\left(A_{D / 16}\right) \leq 2 \mathrm{e}^{-\rho_{0} D^{2} / 1024} .
$$

In both cases,

$$
\rho_{0} D^{2}-C \sqrt{(n-1) K} D-C n \leq 0
$$

for some numerical constant $C>0$. Hence

$$
D \leq \frac{C \sqrt{(n-1) K}+\sqrt{C^{2}(n-1) K+4 C \rho_{0} n}}{2 \rho_{0}}
$$

and thus

$$
D \leq \frac{C \sqrt{(n-1) K}+\sqrt{C \rho_{0} n}}{\rho_{0}}
$$

which yields the conclusion. The theorem is established.

Corollary 4.3. Let $X$ be a compact Riemannian manifold with dimension $n$ and non-negative Ricci curvature. Then

$$
\rho_{0} \leq \frac{C n}{D^{2}}
$$

for some numerical constant $C>0$. 
Corollary 4.3 has to be compared to Cheng's upper bound [Chen] on the spectral gap of compact manifolds with non-negative Ricci curvature

$$
\lambda_{1} \leq \frac{2 n(n+4)}{D^{2}} .
$$

Hence, generically, the difference between the upper bound on $\lambda_{1}$ and $\rho_{0}$ seems to be of the order of $n$. Moreover, it is mentioned in [Chen] that there exist examples with $\lambda_{1} \approx n^{2} / D^{2}$. They indicate that both Rothaus' lower bound (4.4) and Corollary 4.3 could be sharp. Note also that (4.4) together with Corollary 4.3 allows us to recover Cheng's upper bound on $\lambda_{1}$ of the same order in $n$.

Corollary 4.3 is stated for (compact) manifolds without boundary but it also holds for compact manifolds of non-negative Ricci curvature with convex boundary (and Neuman's conditions). In particular, this result applies to convex bounded domains in $\mathbb{R}^{n}$ equipped with normalized Lebesgue measure. If we indeed closely inspect the proof of Theorem 4.2 in the latter case for example, we see that what is only required is (4.5), that holds similarly, and the volume comparisons. These are however well-known and easy to establish for bounded convex domains in $\mathbb{R}^{n}$. In this direction, it might be worthwhile mentioning moreover that the first non-zero Neumann eigenvalue $\lambda_{1}$ of the Laplacian on radial functions on the Euclidean ball $B$ in $\mathbb{R}^{n}$ behaves as $n^{2}$. It may be identified indeed as the square of the first positive zero $\kappa_{n}$ of the Bessel function $J_{n / 2}$ of order $n / 2$ (cf. [Cha1] e.g.). (On a sphere of radius $r$, there will be a factor $r^{-2}$ by homogeneity.) In particular, standard methods or references [Wat] show that $\kappa_{n} \approx n$ as $n$ is large. Denoting by $\rho_{0}$ the logarithmic Sobolev constant on radial functions on $B$, a simple adaption of the proof of Theorem 4.2 shows that $\rho_{0} \leq C n$ for some numerical constant $C>0$. Actually, $\rho_{0}$ is of the order of $n$ and this may be shown directly in dimension one by a simple analysis of the measure with density $n x^{n-1}$ on the interval $[0,1]$. We are indebted to S. Bobkov for this observation. One can further measure on this example the difference between the spectral gap and the logarithmic Sobolev constant as the dimension $n$ is large. (On general functions, $\lambda_{1}$ and $\rho_{0}$ are both of the order of $n$, see [Bo].)

As another application, assume $\operatorname{Ric}_{g} \geq R>0$. As we have seen, by the BakryEmery inequality [B-E], $\rho_{0} \geq R_{n}$ where $R_{n}=\frac{R}{1-\frac{1}{n}}$. Therefore, by Corollary 4.3,

$$
D \leq C \sqrt{\frac{n-1}{R}} .
$$

Up to the numerical constant, this is just Myers' theorem on the diameter of a compact manifold $D \leq \pi \sqrt{\frac{n-1}{R}}$ (cf. [Cha2]). This could suggest that the best numerical constant in Corollary 4.3 is $\pi^{2}$.

Dimension free lower bounds on the logarithmic Sobolev constant in manifolds with non-negative Ricci curvature, similar to the lower bound (3.3) on the spectral gap, are also available. It has been shown by F.-Y. Wang [Wan] (see also [B-L-Q] and [Le3] for slightly improved quantitative estimates) that, if $\operatorname{Ric}_{g} \geq 0$,

$$
\rho_{0} \geq \frac{\lambda_{1}}{1+2 D \sqrt{\lambda_{1}}}
$$

In particular, together with (3.1),

$$
\rho_{0} \geq \frac{\pi^{2}}{(1+2 \pi) D^{2}} .
$$


The preceding lower bound holds more generally for the logarithmic Sobolev constants of Laplace operators with drift $\mathrm{L}=\Delta_{g}-\nabla U \cdot \nabla$ for a smooth function $U$ (with finite, time reversible measure $d \mu=\mathrm{e}^{-U} d v$ ) of non-negative curvature in the sense that, as symmetric tensors,

$$
\operatorname{Ric}_{g}-\nabla \nabla U \geq 0 \text {. }
$$

Under this condition, it is actually shown in [Wan] that if for some $c>0$ and some (all) $x$ in $X$,

$$
\int_{X} \mathrm{e}^{c d(x, \cdot)^{2}} d \mu \leq C<\infty
$$

then $\rho_{0}>0$, with a lower bound depending on $c, C$. In $\mathbb{R}^{n}$ with $U$ convex, S. Bobkov [Bo] showed that $\lambda_{1}>0$ with a lower bound depending on $n$. It would be a challenging question in this context to establish a lower bound on $\lambda_{1}$ only depending on $c, C>0$ such that

$$
\int_{X} \mathrm{e}^{c d(x, \cdot)} d \mu \leq C<\infty .
$$

We refer to the previous references for further details.

Next, we describe analagous results in the discrete case. As in Section 2.1, let $\Pi(x, y)$ be a Markov chain on a finite state space $X$ with symmetric invariant probability measure $\mu$. Let $\rho_{0}$ be the logarithmic Sobolev constant of $(\Pi, \mu)$ defined as the largest $\rho$ such that

$$
\rho \int_{X} f^{2} \log f^{2} d \mu \leq 2 \mathcal{Q}(f, f)
$$

for every $f$ on $X$ with $\int_{X} f^{2} d \mu=1$. Recall that here

$$
\mathcal{Q}(f, f)=\sum_{x, y \in X}[f(x)-f(y)]^{2} \Pi(x, y) \mu(\{x\}) .
$$

Recall also we set

$$
\left\|\left|f \|_{\infty}^{2}=\sup _{x \in X} \sum_{y \in X}\right| f(x)-\left.f(y)\right|^{2} \Pi(x, y)\right.
$$

and denote by $d_{\mathcal{Q}}$ the associated metric.

Arguing as for Theorem 4.1, and using (2.3), we may obtain similarly normal concentration from the logarithmic Sobolev constant $\rho_{0}$.

TheOREM 4.4. Let $(\Pi, \mu)$ be a reversible Markov chain on $X$ as before with logarithmic Sobolev constant $\rho_{0}$. Then

$$
\mathrm{E}_{\left(X, d_{\mathcal{Q}}, \mu\right)}(\lambda) \leq \mathrm{e}^{\lambda^{2} / \rho_{0}}, \quad \lambda \in \mathbb{R} .
$$

In particular $\left(X, d_{\mathcal{Q}}, \mu\right)$ has normal concentration whenever $\rho_{0}>0$.

In the context of Corollary 2.3, we have similarly that whenever $k_{0}=\max \{k(x) ; x \in$ $V\}<\infty$,

$$
\mathrm{E}_{\left(X, d_{c}, \mu\right)}(\lambda) \leq \mathrm{e}^{k_{0} \lambda^{2} / \rho_{0}}, \quad \lambda \in \mathbb{R} .
$$

The next statement is analagous to Proposition 3.2 for the logarithmic Sobolev constant. Denote by $D_{\mathcal{Q}}$ the diameter of $X$ for $d_{\mathcal{Q}}$. The proof is an immediate consequence of Lemma 1.1 and Theorem 4.4. The numerical constant is not sharp. 
Proposition 4.5. If $\mu$ is nearly constant, that is if there exists $C$ such that, for every $x, \mu(\{x\}) \leq C \min _{y \in X} \mu(\{y\})$, then

$$
D_{\mathcal{Q}}^{2} \leq \frac{16 \log (C|X|)}{\rho_{0}}
$$

where $|X|$ is the cardinal of $X$.

As already discussed, the distance most often used in the present setting is not $d$ but the combinatoric distance $d_{c}$ associated with the graph with vertex-set $X$ and edge-set $\{(x, y): \Pi(x, y)>0\}$. Recall that $d_{c} \leq d$ (so that in particular, the combinatoric diameter $D_{c}$ satisfies $D_{c} \leq D_{\mathcal{Q}}$.) Hence, Proposition 4.5 also holds with $D_{c}$.

It is worthwhile mentioning that a small improvement of the concentration bound may be obtained with the graph distance $d_{c}$. Indeed, reproducing the argument leading to (2.3) actually shows that when

$$
\|\nabla f\|_{\infty}=\sup \{|f(x)-f(y)| ; \Pi(x, y)>0\} \leq 1,
$$

for every $\lambda \geq 0$,

$$
\begin{aligned}
\mathcal{Q}\left(\mathrm{e}^{\lambda F / 2}, \mathrm{e}^{\lambda F / 2}\right) & =2 \sum_{F(y)<F(x)}\left(1-\mathrm{e}^{-\lambda[F(x)-F(y)] / 2}\right)^{2} \mathrm{e}^{\lambda F(x)} \Pi(x, y) \mu(\{x\}) \\
& \leq 2\left(1-\mathrm{e}^{-\lambda / 2}\right)^{2} \int_{X} \mathrm{e}^{\lambda F} d \mu \\
& \leq 2 \min \left(1, \frac{\lambda^{2}}{4}\right) \int_{X} \mathrm{e}^{\lambda F} d \mu .
\end{aligned}
$$

The proof of Theorem 4.1 then yields that

$$
\mathrm{E}_{\left(X, d_{c}, \mu\right)}(\lambda) \leq \mathrm{e}^{2 \phi(\lambda) / \rho_{0}}, \quad \lambda \geq 0,
$$

where $\phi(\lambda)=\lambda^{2}$ if $\lambda \leq 2$ and $\phi(\lambda)=4(\lambda-1)$ if $\lambda \geq 2$. Together with Lemma 1.1, we thus draw, under the assumption of Proposition 4.5, an upper bound on $\rho_{0}$ in term of the graph diameter $D_{c}$ as

$$
\rho_{0} \leq \min \left(\frac{8}{D_{c}}, \frac{16 \log (C|X|)}{D_{c}^{2}}\right) .
$$

Results such as Proposition 4.5 and (4.8) may be used as efficient upper bounds on the logarithmic Sobolev constant $\rho_{0}$ in terms of simple geometric objects such as the graph diameter $D_{c}$. These are of interest in the study of rates of convergence to equilibrium for finite Markov chains. While it is classical that the spectral gap $\lambda_{1}$ governs the asymptotic exponential rate of convergence to equilibrium, it has been shown by P. Diaconis and L. Saloff-Coste [SC1], [SC2], [SC3], [D-SC], both in the continuous and discrete cases actually, that the logarithmic Sobolev constant $\rho_{0}$ is more closely related to convergence to stationarity than $\lambda_{1}$ is. Let us now survey a few of examples of interest, kindly communicated to us by L. Saloff-Coste (cf. [D-SC], [SC3] for the necessary background).

Consider first the hypercube $\{0,1\}^{n}$ with $\Pi(x, y)=1 / n$ if $x, y$ differ by exactly one coordinate and $\Pi(x, y)=0$ otherwise. The reversible measure is the uniform distribution and it is classical (see $[\mathrm{D}-\mathrm{SC}]$ ) that $\rho_{0}=4 / n$. The bound (4.8) tells us that $\rho_{0} \leq 8 / n$. 
Consider the Bernoulli-Laplace model of diffusion. This is a Markov chain on the $n$-sets of an $N$-set with $n \leq N / 2$. If the current state is an $n$-set $A$, we pick an element $x$ at random in $A$, an element $y$ at random in the complement $A^{c}$ of $A$ and change $A$ to $B=(A \backslash\{x\}) \cup\{y\}$. The kernel $\Pi$ is given by $\Pi(A, B)=1 /[n(N-n)]$ if $|A \cap B|=n-2$ and $\Pi(A, B)=0$ otherwise. The uniform distribution $\pi(A)=\left(\begin{array}{l}N \\ n\end{array}\right)^{-1}$ is the reversible measure. Clearly, $D_{c}=n$. Hence, by (4.8), $\rho_{0} \leq 8 / n$ which is the right order of magnitude [L-Y].

Let now the random transposition chain on the symmetric group $S_{n}, n \geq 2$. Here, $\Pi(\sigma, \theta)=2 /[n(n-1)]$ if $\theta=\sigma \tau$ for some transposition $\tau$ and $\Pi(\sigma, \theta)=0$ otherwise and $\pi \equiv(n !)^{-1}$, The diameter is $D_{c}=n-1$ and one knows that $\rho_{0}$ is of order $1 / n \log n$ [D-SC], [L-Y]. Here (4.8) only yields $\rho_{0} \leq 16 / n$. Since we know that $\rho_{0} \geq(2 n \log n)^{-1}[\mathrm{D}-\mathrm{SC}]$, we can also conclude from Proposition 4.5 that

$$
D_{\mathcal{Q}} \leq \sqrt{\frac{32 n \log n}{\rho_{0}}} \leq 8 n \log n .
$$

It is not clear whether or not this bound can be obtained more easily. Note that the upper bound

$$
d_{\mathcal{Q}}(\sigma, \theta) \leq\left(\min _{\Pi(\sigma, \theta)>0} \Pi(\sigma, \theta)\right)^{-1 / 2} d_{c}(x, y)
$$

only yields $D_{\mathcal{Q}} \leq n^{2}$, up to a multiplicative constant. It might be worthwhile observing that in this example, $\rho_{0}$ is of order $1 / n \log n$ while it has been shown by B. Maurey $[\mathrm{Ma}]$ that concentration (with respect to the combinatoric metric) is satisfied at a rate of the order of $1 / n$ (see below).

Consider a $N$-regular graph with $N$ fixed. Let $\Pi(x, y)=1 / N$ if they are neighbors and $\Pi(x, y)=0$ otherwise. Then $\mu(\{x\})=1 /|X|$. Assume that for some constant $C>0$, and all $x \in X$ and $t>0$,

$$
|B(x, 2 t)| \leq C|B(x, t)|
$$

where $B(x, t)$ is the ball with center $x$ and radius $t$ in the graph distance $d_{c}$, and $|B(x, t)|$ its number of elements. Fix $x, y \in X$ such that $d_{c}(x, y)=D_{c}$. Set $A=B\left(x, \frac{D_{c}}{2}\right)$ and $B=B\left(y, \frac{D_{c}}{4}\right)$. By (4.10), $\left|B\left(x, \frac{D_{c}}{2}\right)\right| \geq C^{-1}|X|$ and $\left|B\left(y, \frac{D_{c}}{4}\right)\right| \geq$ $C^{-2}|X|$ so that

$$
\mu(A) \geq \frac{1}{C} \quad \text { and } \quad \mu(B) \geq \frac{1}{C^{2}} .
$$

Since $d_{c}(A, B) \geq \frac{D_{c}}{4}$, by Theorem 4.4 and Lemma 1.1 ,

$$
\rho_{0} \leq \frac{192 \log C}{D_{c}^{2}}
$$

For $N$ and $C$ fixed, this is the right order of magnitude in the class of Cayley graphs of finite groups satisfying the volume doubling condition (4.10). See [D-SC, Theorem 4.1].

As a last example, consider any $N$-regular graph on a finite set $X$. Let $\Pi(x, y)=$ $1 / N$ if they are neighbors and $\Pi(x, y)=0$ otherwise. Then $\mu(\{x\})=1 /|X|$ and $|X| \leq N^{D_{c}}$ (at least if $D_{c} \geq 2$ ). From (4.8), $\rho_{0} \leq 8 / D_{c}$. Compare with the results of $[\mathrm{D}-\mathrm{SC}]$ and the Riemannian case. This is, in a sense, optimal generically. Indeed, if $|X| \geq 4$, one also have the lower bound [D-SC]

$$
\rho_{0} \geq \frac{\lambda}{D_{c} \log N}
$$


where $1-\lambda$ is the second largest eigenvalue of $\Pi$. There are many known families of $N$-regular graphs ( $N$ fixed) such that $|X| \rightarrow \infty$ whereas $\lambda \geq \epsilon>0$ stays bounded away from zero (the so-called expanders graphs). Moreover graphs with this property are "generic" amongst $N$-regular graphs [Al].

In the study of birth and death Markov chains, and especially Poisson point processes, some modified versions of the logarithmic Sobolev inequalities have been recently considered $[\mathrm{B}-\mathrm{T}]$. One of them is the entropic inequality that gives rise to the entropic constant $\rho_{1}$ defined as the largest $\rho$ such that

$$
2 \rho \int f \log f d \mu \leq \mathcal{Q}(f, \log f)
$$

when $f$ runs over all finitely supported functions $f: X \rightarrow \mathbb{R}^{+}$such that $\int_{X} f d \mu=1$. The entropic inequality and constant have been considered in $[\mathrm{Wu}]$ for Poisson measure on $\mathbb{N}$, and in the present context in the recent contributions [B-T], [G-Q], [Go]. It is pointed out there that, in general,

$$
\lambda_{1} \geq \rho_{1} \geq \rho_{0}
$$

and that $\rho_{1}$ is also suited to control convergence to equilibrium in the total variation distance. In some typical examples, the entropic constant $\rho_{1}$ however turns out to be a much better rate than the logarithmic Sobolev constant $\rho_{0}$. For example, while on the symmetric discrete cube $\{0,1\}^{n}, \rho_{0}=\rho_{1}=\lambda_{1}=4 / n$, on the cube with weight $p$ and $q(p+q=1), \rho_{1} \sim \lambda_{1}$ but $\rho_{0} \ll \lambda_{1}$ as $p q \rightarrow 0$. Similarly, on the symmetric group $S_{n}$ with the random transposition chain, $\rho_{1} \sim \lambda_{1} \sim 1 / n$ (as $n \rightarrow \infty$ ) (cf. the previous references), while, as mentioned above, $\rho_{0} \sim 1 / n \log n$. With respect to the logarithmic Sobolev constant, the entropic constant seems however inadequate to control convergence in $\ell^{2}$ (cf. [Go]).

Arguing as for the proof of Theorem 4.4, we may get a concentration bound from the entropic inequality. Indeed, as for (2.3), we see that for every $\lambda \geq 0$,

$$
\mathcal{Q}\left(\lambda F, \mathrm{e}^{\lambda F}\right) \leq 2 \lambda^{2}\|\| F \|\left.\right|^{2} \int_{X} \mathrm{e}^{\lambda F} d \mu,
$$

yielding thus Theorem 4.4, and similarly Proposition 4.5, but with the improved entropic constant $\rho_{1} \geq \rho_{0}$. In particular, since $\rho_{1} \geq 1 / 2(n-1)$ on $S_{n}$, we recover Maurey's concentration result [Ma]. Furthermore,

$$
D_{\mathcal{Q}} \leq 8 n \sqrt{\log n}
$$

that improves upon (4.9).

Again, the graph distance $d_{c}$ yields some improved bounds (cf. [B-T], [Go]). Namely, arguing as for (4.7), we have that, for every $\lambda \geq 0$ and every $F$ with $\|\nabla F\|_{\infty} \leq 1$

$$
\mathcal{Q}\left(\lambda F, \mathrm{e}^{\lambda F}\right) \leq 2 \lambda\left(1-\mathrm{e}^{-\lambda}\right) \int_{X} \mathrm{e}^{\lambda F} d \mu \leq 2 \min \left(\lambda, \lambda^{2}\right) \int_{X} \mathrm{e}^{\lambda F} d \mu .
$$

The Herbst argument then applies to yield the following consequence.

TheOREM 4.6. Let $(\Pi, \mu)$ be a reversible Markov chain on $X$ as before with entropic constant $\rho_{1}$. Then

$$
\mathrm{E}_{\left(X, d_{c}, \mu\right)}(\lambda) \leq \mathrm{e}^{\min \left(\lambda^{2}, \lambda\left(1+\log ^{+} \lambda\right)\right) / \rho_{1}}, \quad \lambda \geq 0 .
$$


By Lemma 1.1, we deduce from Theorem 4.6 a bound, to be compared to (4.8), on the entropic constant $\rho_{1}$ in terms of the graph diameter $D_{c}$, namely

$$
\rho_{1} \leq \min \left(\frac{2}{D_{c}} \log \left(\frac{2 \mathrm{e}^{2}}{D_{c}} \log (C|X|) \vee \mathrm{e}\right), \frac{16 \log (C|X|)}{D_{c}^{2}}\right) .
$$

The previous Markov chains examples may then be analyzed via the entropic constant $\rho_{1}$ to yield, depending on the cases, possibly sharper bounds.

We refer to $[\mathrm{B}-\mathrm{T}],[\mathrm{G}-\mathrm{Q}],[\mathrm{Go}]$ for further results and details on the entropic constant.

\section{Dimension free isoperimetric bounds}

In this last section, we investigate some inequalities of isoperimetric type that may be produced from spectral and logarithmic Sobolev informations. While sharper dimensional bounds are known and classical, we emphasize here dimension free estimates of interest in the study of diffusion operators with drifts (cf. [Ba], [Le4]). The result improve upon [Le1] (see also [Ba-L]).

Let $(X, g)$ be a smooth complete connected Riemanian manifold, and let $\Delta_{g}$ be the Laplace operator on $(X, g)$. Let $\left(P_{t}\right)_{t \geq 0}$ be the heat semigroup (cf. [Da]). It is worthwhile mentioning that, whenever $(\bar{X}, g)$ is of finite volume $V$, both the spectral gap $\lambda_{1}$ and logarithmic Sobolev constants admit equivalent description in terms of smoothing properties of $\left(P_{t}\right)_{t \geq 0}$. Denote by $d \mu=\frac{d v}{V}$ the normalized volume element. By the spectral theorem

$$
\left\|P_{t} f\right\|_{2} \leq \mathrm{e}^{-\lambda_{1} t}\|f\|_{2}, \quad t \geq 0
$$

for every $f$ with $\int_{X} f d \mu=0$, where $\|\cdot\|_{p}$ is the $\mathrm{L}^{p}$-norm $(1 \leq p \leq \infty)$ with respect to $\mu$. A fundamental theorem of L. Gross [Gros] shows that $\rho_{0}$ may be characterized by the hypercontractivity property

$$
\left\|P_{t} f\right\|_{q} \leq\|f\|_{p}
$$

for every $f$ whenever $1<p<q<\infty$ and $\mathrm{e}^{\rho_{0} t} \geq[(q-1) /(p-1)]^{1 / 2}$.

The next lemma is a reversed Poincaré inequality for heat kernel measures (cf. [Le4]). We use it below as a weak, dimension free, form of the Li-Yau parabolic gradient inequality [L-Y2].

Lemma 5.1. Assume that $\mathrm{Ric}_{g} \geq-K, K \geq 0$. Then, for every $t \geq 0$ and every smooth function $f$ on $(X, g)$, at every point,

$$
c(t)\left|\nabla P_{t} f\right|^{2} \leq P_{t}\left(f^{2}\right)-\left(P_{t} f\right)^{2}
$$

where

$$
c(t)=\frac{1-\mathrm{e}^{-2 K t}}{K} \quad(=2 t \text { if } K=0) .
$$

Proof. For a smooth function $f$ on $(X, g)$, and $t>0$ fixed, set

$$
\varphi(s)=\mathrm{e}^{2 K s} P_{s}\left(\left|\nabla P_{t-s} f\right|^{2}\right), 0 \leq s \leq t
$$

(evaluated at some point in $X$ ). By the chain rule for differentiation,

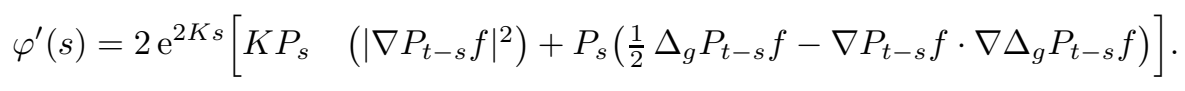

By the Bochner formula,

$$
\frac{1}{2} \Delta_{g} P_{t-s} f-\nabla P_{t-s} f \cdot \nabla \Delta_{g} P_{t-s} f \geq-K\left|\nabla P_{t-s} f\right|^{2} .
$$


Hence $\varphi$ is non-decreasing so that, for every $t \geq 0$, and at every point,

$$
\left|\nabla P_{t} f\right|^{2} \leq \mathrm{e}^{2 K t} P_{t}\left(|\nabla f|^{2}\right) \text {. }
$$

Write then

$$
P_{t}\left(f^{2}\right)-\left(P_{t} f\right)^{2}=\int_{0}^{t} \frac{d}{d s} P_{s}\left(\left(P_{t-s} f\right)^{2}\right) d s=2 \int_{0}^{t} P_{s}\left(\left|\nabla P_{t-s} f\right|^{2}\right) d s .
$$

By (5.3), $P_{s}\left(\left|\nabla P_{t-s} f\right|^{2}\right) \geq \mathrm{e}^{-2 K s}\left|\nabla P_{t} f\right|^{2}$ so that the claim follows. The proof is complete.

As a consequence of this lemma, and since $1-\mathrm{e}^{-u} \geq \frac{u}{2}$ for every $0 \leq u \leq 1$, note that for every $0<t \leq \frac{1}{2 K}$, and every smooth bounded function $f$ on $(X, g)$,

$$
\left\|\nabla P_{t} f\right\|_{\infty} \leq \frac{1}{\sqrt{t}}\|f\|_{\infty} .
$$

In particular, integrating (5.4) yields, by duality, that for every smooth function $f$ and every $0<t \leq \frac{1}{2 K}$,

$$
\left\|f-P_{t} f\right\|_{1} \leq 2 \sqrt{t}\|\nabla f\|_{1} .
$$

Indeed, for every $g$ smooth with $\|g\|_{\infty} \leq 1$,

$$
\begin{aligned}
\int_{X} g\left(f-P_{t} f\right) d \mu & =-\int_{0}^{t}\left(\int_{X} g \Delta_{g} P_{s} f d \mu\right) d s \\
& =\int_{0}^{t}\left(\int_{X} \nabla P_{s} g \cdot \nabla f d \mu\right) d s \\
& \leq\|\nabla f\|_{1} \int_{0}^{t}\left\|\nabla P_{s} g\right\|_{\infty} d s \\
& \leq 2 \sqrt{t}\|\nabla f\|_{1}
\end{aligned}
$$

and the claim follows.

Provided with this result, the next theorems describe isoperimetric type bounds under spectral and logarithmic Sobolev constants. If $A$ is an open subset of $X$ with smooth boundary $\partial A$, we denote by $\mu_{s}(\partial A)$ the surface measure of $\partial A$.

THEOREM 5.2. Let $(X, g)$ be a smooth complete connected Riemannian manifold without boundary with finite volume $V$, and denote by $d \mu=\frac{d v}{V}$ the normalized volume element. Assume that $\mathrm{Ric}_{g} \geq-K, K \geq 0$. Then, if $\lambda_{1}$ denotes the first non-trivial eigenvalue of $\Delta_{g}$ on $(X, g)$, for any open subset $A$ of $X$ with smooth boundary $\partial A$,

$$
\mu_{s}(\partial A) \geq \frac{1}{3} \min \left(\frac{\lambda_{1}}{\sqrt{K}}, \sqrt{\lambda_{1}}\right) \mu(A)(1-\mu(A)) .
$$

Theorem 5.2 produces equivalently a lower bound on the Cheeger constant defined as the largest $h$ such that

$$
\mu_{s}(\partial A) \geq h \min (\mu(A), 1-\mu(A))
$$

for all open subsets $A$ of $X$ with smooth boundary $\partial A$. Recall from [Chee] that $h \leq 2 \sqrt{\lambda_{1}}$. In this form, Theorem 5.2 goes back to the work by P. Buser [Bu]. It is a remarkable fact however that Theorem 5.2 yields constants independent of the dimension of the manifold. 
ProOF. We apply (5.5) to smooth functions approximating the characteristic function $\chi_{A}$ of an open set $A$ in $X$ with smooth boundary $\partial A$. It yields, for every $0<t \leq \frac{1}{2 K}$,

$$
\begin{aligned}
2 \sqrt{t} \mu_{s}(\partial A) & \geq \int_{A}\left[1-P_{t}\left(\chi_{A}\right)\right] d \mu+\int_{A^{c}} P_{t}\left(\chi_{A}\right) d \mu \\
& =2\left(\mu(A)-\int_{A} P_{t}\left(\chi_{A}\right) d \mu\right) \\
& =2\left(\mu(A)-\left\|P_{t / 2}\left(\chi_{A}\right)\right\|_{2}^{2}\right)
\end{aligned}
$$

where we used reversibility of the heat semigroup $\left(P_{t}\right)_{t \geq 0}$ with respect to the Riemannian volume element (cf. [Da]). Now, by (5.1),

$$
\begin{aligned}
\left\|P_{t / 2}\left(\chi_{A}\right)\right\|_{2}^{2} & =\mu(A)^{2}+\left\|P_{t / 2}\left(\chi_{A}-\mu(A)\right)\right\|_{2}^{2} \\
& \leq \mu(A)^{2}+\mathrm{e}^{-\lambda_{1} t}\left\|\chi_{A}-\mu(A)\right\|_{2}^{2}
\end{aligned}
$$

so that, with the preceding,

$$
\sqrt{t} \mu_{s}(\partial A) \geq \mu(A)(1-\mu(A))\left(1-\mathrm{e}^{-\lambda_{1} t}\right)
$$

for every $0<t \leq \frac{1}{2 K}$. We need simply optimize in $0<t \leq \frac{1}{2 K}$ to conclude: if $\lambda_{1} \geq 2 K$, we can choose $t=\frac{1}{\lambda_{1}}$ while if $\lambda_{1} \leq 2 K$, we simply take $t=\frac{1}{2 K}$. The result follows.

The next statement is the corresponding result for the logarithmic Sobolev constant. The numerical constant is not sharp.

TheOREM 5.3. Let $(X, g)$ be a smooth complete connected Riemannian manifold without boundary with finite volume $V$, and denote by $d \mu=\frac{d v}{V}$ the normalized volume element. Assume that $\operatorname{Ric}_{g} \geq-K, K \geq 0$. Then, if $\rho_{0}$ denotes the logarithmic Sobolev constant of $\Delta_{g}$ on $(X, g)$, for any open subset $A$ of $X$ with smooth boundary $\partial A$ such that $\mu(A) \leq \frac{1}{2}$,

$$
\mu_{s}(\partial A) \geq \frac{1}{34} \min \left(\frac{\rho_{0}}{\sqrt{K}}, \sqrt{\rho_{0}}\right) \mu(A)\left(\log \frac{1}{\mu(A)}\right)^{1 / 2} .
$$

PROOF. We follow the proof of Theorem 5.2 using now that $\rho_{0}$ is characterized by the hypercontractivity property (5.2). As we have seen in the proof of Theorem 5.2 , for every open set $A$ in $X$ with smooth boundary $\partial A$, and every $0<t \leq \frac{1}{2 K}$,

$$
\sqrt{t} \mu_{s}(\partial A) \geq \mu(A)-\left\|P_{t / 2}\left(\chi_{A}\right)\right\|_{2}^{2} .
$$

Now, by (5.2) with $p=2$ and $q=1+\mathrm{e}^{-\rho_{0} t}$,

$$
\left\|P_{t / 2}\left(\chi_{A}\right)\right\|_{2}^{2} \leq \mu(A)^{2 /\left(1+\mathrm{e}^{-\rho_{0} t}\right)} .
$$

Since $1-\mathrm{e}^{-u} \geq \frac{u}{2}, 0 \leq u \leq 1$, it follows that

$$
\sqrt{t} \mu_{s}(\partial A) \geq \mu(A)\left[1-\exp \left(-\frac{\rho_{0} t}{4} \log \frac{1}{\mu(A)}\right)\right] .
$$

Set $t_{0}=\min \left(\frac{1}{2 K}, \frac{1}{\rho_{0}}\right)$. Choose then $0<t \leq t_{0}$ such that

$$
t=4 t_{0}\left(\log \frac{1}{\mu(A)}\right)^{-1}
$$


provided $\mu(A)$ is small enough so that $\mu(A) \leq \mathrm{e}^{-4}$. For this value of $t,(5.7)$ yields

$$
\begin{aligned}
\mu_{s}(\partial A) & \geq \frac{1}{2 \sqrt{t_{0}}}\left(1-\mathrm{e}^{-\rho_{0} t_{0}}\right) \mu(A)\left(\log \frac{1}{\mu(A)}\right)^{1 / 2} \\
& \geq \frac{1}{4} \rho_{0} \sqrt{t_{0}} \mu(A)\left(\log \frac{1}{\mu(A)}\right)^{1 / 2}
\end{aligned}
$$

since $\rho_{0} t_{0} \leq 1$. This inequality holds for $\mu(A) \leq \mathrm{e}^{-4}$. In general however, when $0 \leq \mu(A) \leq \frac{1}{2}$, we can always apply (5.7) with $t=t_{0}$ to get

$$
\mu_{s}(\partial A) \geq \frac{1}{\sqrt{t_{0}}} \mu(A)\left[1-\exp \left(-\frac{\rho_{0} t_{0}}{4} \log 2\right)\right] \geq \frac{1}{12} \rho_{0} \sqrt{t_{0}} \mu(A) .
$$

Combined with the preceding, Theorem 5.3 is established.

The preceding results hold, with the same proofs, in the context of diffusion operators $\Delta_{g}-\nabla U \cdot \nabla$ with non-negative curvature in the sense that

$$
\operatorname{Ric}_{g}-\nabla \nabla U \geq 0
$$

(cf. [Ba-L]). In particular, if $\mu$ is a log-concave probability measure on $\mathbb{R}^{n}$, its Cheeger constant $h$ (of (5.6)) and Poincaré constant $\lambda_{1}$ satisfy

$$
\frac{1}{6} \sqrt{\lambda_{1}} \leq h \leq 2 \sqrt{\lambda_{1}}
$$

A deep conjecture of R. Kannan, L. Lovàsz and M. Simonovits [K-L-S] asserts that the Cheeger constant $h$ should be bounded below by a universal strictly positive constant in the class of all log-concave probability measures $\mu$ under the isotropic condition

$$
\int_{\mathbb{R}^{n}}\langle x, \theta\rangle^{2} d \mu(x)=|\theta|^{2} \quad \text { for all } \quad \theta \in \mathbb{R}^{n} .
$$

By (5.8), the question is thus reduced to the corresponding one for the easier Poincaré constant.

Discrete versions of Theorems 5.2 and 5.3 are studied in [B-H-T] and [H-T].

While only of logarithmic type with respect to the (power type) isoperimetric comparison theorems of M. Gromov [Grom1] (cf. [Grom2]) and [B-B-G], the isoperimetric bound of Theorem 5.3, on the other hand, involves $\rho_{0}$ rather than the diameter of the manifold, and is independent of the dimension of the manifold (dimension is actually hidden in Theorem 4.2). In the context of diffusion operators of the preceding type, this information is a weaker one since (in contrast with the Sobolev constants) the hypercontractivity constant does not usually control the diameter of the manifold, as is shown by the example of $\Delta-x \cdot \nabla$ on $\mathbb{R}^{n}$ (with the standard Gaussian measure as invariant measure). Actually, the isoperimetric function in Theorem 5.3 is a form of the isoperimetric function in Gauss space (cf. [Le4], [Le5]) for which the "infinite dimensional" extension of the Lévy-Gromov isoperimetric inequality of [Grom1 (cf. [Grom2]) is studied in [Ba-L].

Acknowledgement. Thanks are due to Professors A. Grigor'yan and S.T. Yau for their invitation to write this paper. We also thank L. Saloff-Coste for several comments, years ago, about the subject of these notes, and S. Bobkov for several precious observations. 


\section{REFERENCES}

[A-M-S] S. Aida, T. Masuda, I. Shigekawa. Logarithmic Sobolev inequalities and exponential integrability. J. Funct. Anal. 126, 83-101 (1994).

[A-S] S. Aida, D. Stroock. Moment estimates derived from Poincaré and logarithmic Sobolev inequalities. Math. Res. Lett. 1, 75-86 (1994).

[Al] N. Alon. Eigenvalues and expanders. J. Combin. Theory, Ser. B, 38, 78-88 (1987).

[A-M] N. Alon, V. Milman. $\lambda_{1}$, isoperimetric inequalities for graphs ans superconcentrators. J. Combin. Theory, Ser. B, 38, 78-88 (1985).

[Ba] D. BAKRY. L'hypercontractivité et son utilisation en théorie des semigroupes. Ecole d'Eté de Probabilités de St-Flour. Lecture Notes in Math. 1581, 1-114 (1994). Springer.

[B-E] D. Bakry, M. Emery. Diffusions hypercontractives. Séminaire de Probabilités XIX. Lecture Notes in Math. 1123, 177-206 (1985). Springer.

[Ba-L] D. BAKry, M. Ledoux. Lévy-Gromov's isoperimetric inequality for an infinite dimensional diffusion generator. Invent. math. 123, 259-281 (1996).

[B-L-Q] D. Bakry, M. Ledoux, Z. Qian. Unpublished manuscript (1997).

[B-B-G] P. BÉrard, G. Besson, S. Gallot. Sur une inégalité isopérimétrique qui généralise celle de Paul Lévy-Gromov. Invent. math. 80, 295-308 (1985).

[Bo] S. BobKov. Isoperimetric and analytic inequalities for log-concave probability measures. Ann. Probab., 27, 1903-1921 (1999).

[B-H-T] S. Bobkov, C. Houdré, P. Tetali. $\lambda_{\infty}$, vertex isoperimetry and concentration. Combinatorica 20, 153-172 (2000).

[Bo-L] S. Bobkov, M. Ledoux. Poincaré's inequalities and Talagrand's concentration phenomenon for the exponential measure. Probab. Theory Relat. Fields 107, 383-400 (1997).

[B-T] S. Bobkov, P. Tetali. Modified logarithmic Sobolev inequalities in discrete settings (2003).

[B-U] A. Borovkov, S. Utev. On a inequality and a related characterization of the normal distribution. Theor. Probab. Appl. 28, 209-218 (1983).

[Br] R. Brooks. On the spectrum of non-compact manifolds with finite volume. Math. Z. 187, 425-437 (1984).

[Bu] P. Buser. A note on the isoperimetric constant. Ann. scient. Éc. Norm. Sup. 15, 213-230 (1982).

[Cha1] I. Chavel. Eigenvalues in Riemannian geometry. Academic Press (1984).

[Cha2] I. Chavel. Riemannian geometry - A modern introduction. Cambridge Univ. Press (1993).

[Chee] J. CheEger. A lower bound for the smallest eigenvalue of the Laplacian. Problems in Analysis, Symposium in honor of S. Bochner. Princeton Univ. Press., 195-199. Princeton (1970).

[C-E] J. Cheeger, D. Ebin. Comparison theorems in Riemannian geometry. North-Holland (1975).

[Chen] S.-Y. Cheng. Eigenvalue comparison theorems and its geometric applications. Math. Z. 143, 289-297 (1975).

[Chu] F. R. K. Chung. Diameters and eigenvalues. J. Amer. Math. Soc. 2, 187-196 (1989).

[C-G-Y1] F. R. K. Chung, A. Grigor'yan, S.-T. Yau. Upper bounds for eigenvalues of tha discrete and continuous Laplace operators. Advances in Math. 117, 165-178 (1996).

[C-G-Y2] F. R. K. Chung, A. Grigor'yan, S.-T. Yau. Eigenvalues and diameters for manifolds and graphs. Tsing Hua lectures on geometry and \& analysis (1990/91) 79-105. Internat. Press Cambridge MA (1997).

[Da] E. B. Davies. Heat kernel and spectral theory. Cambridge Univ. Press (1989).

[Da-S] E. B. DAvies, B. Simon. Ultracontractivity and the heat kernel for Schrödinger operators and Dirichlet Laplacians. J. Funct. Anal. 59, 335-395 (1984).

[De-S] J.-D. Deuschel, D. Stroock. Large deviations. Academic Press (1989).

[D-SC] P. Diaconis, L. Saloff-Coste. Logarithmic Sobolev inequalities for finite Markov chains. Ann. Appl. Probab. 6, 695-750 (1996).

[G-H-L] S. Gallot, D. Hulin, J. Lafontaine. Riemannian Geometry. Second Edition. Springer (1990).

[G-Q] F. Gao, J. Quastel. Exponential decay of entropy in random transposition and Bernoulli-Laplace models (2002). 
[Go] S. GoEL. Modified logarithmic Sobolev inequalities for some models of rnadom walk (2003).

[Grom1] M. Gromov. Paul Lévy's isoperimetric inequality. Preprint I.H.E.S. (1980).

[Grom2] M. Gromov. Metric structures for Riemannian and non-Riemannian spaces. Birkhäuser (1998).

[G-M] M. Gromov, V. D. Milman. A topological application of the isoperimetric inequality. Amer. J. Math. 105, 843-854 (1983).

[Gros] L. Gross. Logarithmic Sobolev inequalities. Amer. J. Math. 97, 1061-1083 (1975).

[H-T] C. Houdré, P. Tetali. Concentration of measure for products of Markov kernels via functional inequalities. Combin. Probab. Comput. 10 1-28 (2001).

[Le1] M. Ledoux. A simple analytic proof of an inequality by P. Buser. Proc. Amer. Math. Soc. 121, 951-959 (1994).

[Le2] M. Ledoux. Remarks on logarithmic Sobolev constants, exponential integrability and bounds on the diameter. J. Math. Kyoto Univ. 35, 211-220 (1995).

[Le3] M. Ledoux. Concentration of measure and logarithmic Sobolev inequalities. Séminaire de Probabilités XXXIII. Lecture Notes in Math. 1709, 120-216 (1999). Springer.

[Le4] M. Ledoux. The geometry of Markov diffusion generators. Ann. Fac. Sci. Toulouse IX, 305-366 (2000).

[Le5] M. Ledoux. The concentration of measure phenomenon. Math. Surveys and Monographs 89. Amer. Math. Soc. (2001).

[L-Y] T.Y. LeE, H.-T. YAU. Logarithmic Sobolev inequality fo some models of random walks. Ann. Probab. 26, 1855-1873 (1998).

[Li] P. Li. A lower bound for the first eigenvalue of the Laplacian on a compact manifold. Indiana Univ. Math. J. 28, 1013-1019 (1979).

[L-Y1] P. LI, S.-T. YAU. Estimates of eigenvalues of a compact Riemannian manifold. Proc. Symp. Pure Math. 36, 205-239. Amer. Math. Soc. (1980).

[L-Y2] P. Li, S.-T. YAu. On the parabolic kernel of the Schrödinger operator. Acta Math. 156, 153-201 (1986).

[Ma] B. Maurey. Constructions de suites symétriques. C. R. Acad. Sci. Paris 288, 679-681 (1979).

[Ro1] O. Rothaus. Diffusion on compact Riemannian manifolds and logarithmic Sobolev inequalities. J. Funct. Anal. 42, 358-367 (1981).

[Ro2] O. Rothaus. Hypercontractivity and the Bakry-Emery criterion for compact Lie groups. J. Funct. Anal. 65, 358-367 (1986).

[SC1] L. Saloff-Coste. Precise estimates on the rate at which certain diffusions tend to equilibrium. Math. Z. 217, 641-677 (1994).

[SC2] L. SAloff-Coste. Convergence to equilibrium and logarithmic Sobolev constant on manifolds with Ricci curvature bounded below. Colloquium Math. 67, 109-121 (1994).

[SC3] L. SAloff-Coste. Lectures on finite Markov chains. Ecole d'Eté de Probabilités de St-Flour 1996. Lecture Notes in Math. 1665, 301-413 (1997). Springer-Verlag.

[Sc] M. Schmuckenschläger. Martingales, Poincaré type inequalities and deviations inequalities. J. Funct. Anal. 155, 303-323 (1998).

[Wan] F.-Y. WANG. Logarithmic Sobolev inequalities on noncompact Riemannian manifolds. Probab. Theory Relat. Fields 109, 417-424 (1997).

[Wat] G. N. Watson. A treatise on the theory of Bessel functions. Cambridge Univ. Press (1944).

[Ya] S.-T. YAU. Isoperimetric constants and the first eigenvalue of a compact Riemannian manifold. Ann. scient. Éc. Norm. Sup. 8, 487-507 (1975).

[Z-Y] J. Q. Zhong, H. C. YAng On the estimate of the first eigenvalue of a compact Riemanian manifold. Sci. Sinica Ser. A 27 (12), 1265-1273 (1984).

Institut de Mathématiques, Université Paul-Sabatier, 31062 Toulouse, France 